\title{
ACCESSIBLE POINTS OF HEREDITARILY DECOMPOSABLE CHAINABLE CONTINUA
}

\author{
PIOTR MINC AND W. R. R. TRANSUE
}

\begin{abstract}
In this paper it is proven that a chainable continuum $X$ can be embedded in the plane in such a way that every point is accessible from its complement if and only if it is Suslinean. An example is shown of an hereditarily decomposable chainable continuum which cannot be embedded in the plane in such a way that each endpoint is accessible.
\end{abstract}

\section{INTRODUCTION}

H. Cook, in the University of Houston problem book, asks (Problem 5) "Can every hereditarily decomposable chainable continuum be embedded in the plane in such a way that each endpoint is accessible from the complement?", and (Problem 6) "Is it true that a chainable continuum can be embedded in the plane in such a way that every point is accessible from the complement if and only if it is Suslinean?". These questions are answered, the first negatively and the second affirmatively. We prove in addition that if the set of nondegenerate generalized end layers (see below for definitions) of an hereditarily decomposable chainable continuum is countable then it may be embedded so that each endpoint is accessible.

A continuum is a compact connected metric space. A continuum is chainable if, for each $\varepsilon>0$ there is an $\varepsilon$-map (i.e. a continuous function whose point inverses have diameter less than $\varepsilon$ ) onto an interval. This notion may be equivalently defined using chain coverings or inverse limits of arcs. A point $x$ of a chainable continuum is an endpoint if, for each $\varepsilon>0$ there is an $\varepsilon$-map onto an interval sending $x$ to an endpoint of the interval. A continuum is decomposable if it can be written as the union of two of its proper subcontinua, otherwise it is indecomposable. A continuum is hereditarily decomposable if each nondegenerate subcontinuum is decomposable. A continuum is Suslinean if any disjoint collection of its nondegenerate subcontinua is countable. Note that each Suslinean continuum is hereditarily decomposable.

Received by the editors May 12, 1990.

1980 Mathematics Subject Classification (1985 Revision). Primary 54F15.

Key words and phrases. Accessible points, hereditarily decomposable, chainable continuum, Suslinean continuum. 
A point $p$ in a subset $X$ of the plane is accessible from the complement provided there is an arc $A$ in the plane so that $X \cap A=\{p\} . \operatorname{Acc}(X)$ is the set of all such points.

Chainable continua can always be embedded in the plane. Indecomposable continua, when embedded in the plane, necessarily leave many points inaccessible from the complement [6]. Hereditarily decomposable chainable continua may often be embedded so that some points are inaccessible (as in a ray spiraling around an arc) or all points are accessible (as in the standard $\sin \frac{1}{x}$ curve). This paper describes a construction which may be viewed as the process of obtaining the latter type of embedding.

\section{Preliminaries}

If $S$ is a set, $\operatorname{cl}(S), \operatorname{int}(S)$ and $\operatorname{int}_{C}(S)$ denote the closure, interior and the interior relative to $C$, respectively. The distance from $x$ to $y$ will be denoted by $|x-y|$. If $\varepsilon>0, \mathscr{B}(S, \varepsilon)=\{x|| x-s \mid<\varepsilon$ for some $s$ in $S\}$. The interior of an arc is the arc minus its endpoints. If $S_{1}, S_{2}, \ldots$ is a sequence of compact sets $\lim _{i \rightarrow \infty} S_{i}$ denotes the limit in the Hausdorff distance [4].

We shall assume some familiarity with Chapter V, $\S 48$ of [4]. In particular given a chainable hereditarily decomposable continuum $X$ (or more generally an hereditarily decomposable continuum irreducible between two points), there is a continuous function $g$ from $X$ onto $[0,1]$ so that $g^{-1}(t)$ is a maximal nowhere dense subcontinuum for each $t$ in $[0,1]$. We shall call $g$ a Kuratowski function. Subcontinua $g^{-1}(t)$ are called layers of $X ; g^{-1}(0)$ and $g^{-1}(1)$ are called end layers of $X$; the other layers are called interior layers. If $x$ and $y$ are points of $X$ we denote by $[x, y]$ the continuum irreducible between $x$ and $y$. Denote $[x, y]$ minus its end layers by $(x, y)$. Denote also $[x, y]$ minus its layer containing $y$ (or $x$ ) by $[x, y)$ (or $(x, y]$, respectively).

We will define layers in a more general sense considering layers of layers and their layers and so on, as in [10 and 8].

Define $\mathscr{L}_{0}$ to be $\{X\}$. If $\alpha=\beta+1$ then $\mathscr{L}_{\alpha}$ will consist of the degenerate elements (points) of $\mathscr{L}_{\beta}$ and the layers of nondegenerate elements of $\mathscr{L}_{\beta}$. For a limit ordinal $\alpha$ define $\mathscr{L}_{\alpha}$ to be the coarsest decomposition of $X$ refining all $\mathscr{L}_{\beta}$ 's for $\beta<\alpha$. Observe that in the last case $\mathscr{L}_{\alpha}$ consists of the intersections $\bigcap_{\beta<\alpha} L_{\beta}$, where $L_{\beta} \in \mathscr{L}_{\beta}$. It was shown in [8] that there is a countable $\tau$ so that $\mathscr{L}_{\tau}$ contains only degenerate elements. The elements of $\bigcup_{\beta \leq \tau} \mathscr{L}_{\beta}$ will be called generalized layers of $X$. By generalized end layers we will understand those generalized layers of $X$ which are not contained in interior layers of any other generalized layer of $X$. (It follows that $X$ itself is a generalized end layer.) Note that any endpoint of $X$ is a degenerate generalized end layer. It should be observed that generalized layers are terminal subcontinua (in the sense of [2]) of $X$. So it follows from [2, Lemma 2, p. 464] that each degenerate generalized end layer is an endpoint of $X$.

By considering Kuratowski functions on generalized layers we can introduce an order (nonunique) " $<$ " on $X$ so that if $a<c<b$ then $c \in[a, b]$, see $[7, \S 3$, p. 178] for details. It is easy to see that if $C$ is a subcontinuum it has leftmost (least) and rightmost (greatest) points which we denote by $l(C)$ and $r(C)$, respectively. 


\section{AN HEREDITARILY DECOMPOSABLE CHAINABLE CONTINUUM}

\section{HAVING UNCOUNTABLY MANY NONDEGENERATE GENERALIZED END LAYERS}

This continuum will be shown (see Corollary 6.6) to be a counterexample to Cook's problem 5. It is helpful to bear this in mind going through $\S \S 4$ and 5 of this paper.

Example 3.1. We will give an example of an hereditarily decomposable continuum $X$ so that for each embedding of $X$ into the plane there will be an endpoint of $X$ which is not accessible from the complement of $X$. Our continuum $X$ is the inverse limit of $[0,1]$ with the bonding maps $f_{0}, f_{1}, f_{2}, \ldots$ defined by the following formulas

$$
f_{0}(t)= \begin{cases}3 t & \text { if } 0 \leq t \leq 1 / 3 \\ 2-3 t & \text { if } 1 / 3 \leq t \leq 2 / 3 \\ 3 t-2 & \text { if } 2 / 3 \leq t \leq 1\end{cases}
$$

and

$$
f_{n+1}(t)= \begin{cases}\frac{1}{3} f_{n}(3 t) & \text { if } 0 \leq t \leq \frac{1}{3} \\ \frac{4}{3}-3 t & \text { if } \frac{1}{3} \leq t \leq \frac{4}{9} \\ 9 t-4 & \text { if } \frac{4}{9} \leq t \leq \frac{5}{9} \\ \frac{8}{3}-3 t & \text { if } \frac{5}{9} \leq t \leq \frac{2}{3} \\ \frac{2}{3}+\frac{1}{3} f_{n}(3 t-2) & \text { if } \frac{2}{3} \leq t \leq 1\end{cases}
$$

Let $p_{n}$ denote the projection of $X$ to $[0,1]$ so that $p_{n}=f_{n} \circ p_{n+1}$ for $n=0,1, \ldots$.

Let $g_{0}:[0,1] \rightarrow[0,1 / 3]$ be defined by $g_{0}(t)=t / 3$ and let $g_{1}:[0,1] \rightarrow$ $[2 / 3,1]$ be defined by $g_{1}(t)=2 / 3+t / 3$. Observe that $g_{i}$ is a homeomorphism and $g_{i} \circ f_{n}=f_{n+1} \circ g_{i}$ for $i=0,1$. So $g_{i}$ induces an embedding $h_{i}$ of $X$ into itself. Observe that $p_{n} \circ h_{i}=g_{i} \circ p_{n}$ for $i=0,1$ and $n=0,1, \ldots$ Let $A_{i}$ denote the continuum $h_{i}(X)$. Observe that $A_{0}$ and $A_{1}$ are the end layers of $X$ and $X \backslash\left(A_{0} \cup A_{1}\right)$ is homeomorphic to the real line.

For each finite sequence $\varepsilon_{0}, \varepsilon_{1}, \ldots, \varepsilon_{n}$ of 0 's and 1's we will define an interval $I\left(\varepsilon_{0}, \varepsilon_{1}, \ldots, \varepsilon_{n}\right)$ by recursion, setting $I(0)=[0,1 / 3], I(1)=[2 / 3,1]$ and $I\left(i, \varepsilon_{1}, \varepsilon_{2}, \ldots, \varepsilon_{n}\right)=g_{i}\left(I\left(\varepsilon_{1}, \varepsilon_{2}, \ldots, \varepsilon_{n}\right)\right)$. Observe that $I\left(\varepsilon_{0}, \varepsilon_{1}, \ldots, \varepsilon_{n}\right)$ $\subset I\left(\varepsilon_{0}, \varepsilon_{1}, \ldots, \varepsilon_{n-1}\right)$ and that $f_{n}$ restricted to $I\left(\varepsilon_{0}, \varepsilon_{1}, \ldots, \varepsilon_{n}\right)$ is a homeomorphism onto $I\left(\varepsilon_{0}, \varepsilon_{1}, \ldots, \varepsilon_{n-1}\right)$ for $n=1,2, \ldots$.

For each finite sequence $\varepsilon_{0}, \varepsilon_{1}, \ldots, \varepsilon_{n}$ of 0 's and 1's we will define a continuum $A\left(\varepsilon_{0}, \varepsilon_{1}, \ldots, \varepsilon_{n}\right)$ by recursion, setting $A(i)=A_{i}$ and

$$
A\left(i, \varepsilon_{1}, \varepsilon_{2}, \ldots, \varepsilon_{n}\right)=h_{i}\left(A\left(\varepsilon_{1}, \varepsilon_{2}, \ldots, \varepsilon_{n}\right)\right) .
$$

Observe that $p_{k}\left(A\left(\varepsilon_{0}, \varepsilon_{1}, \ldots, \varepsilon_{n}\right)\right)=I\left(\varepsilon_{0}, \varepsilon_{1}, \ldots, \varepsilon_{n}\right)$ for $k, n=0,1,2, \ldots$

Let $\varepsilon_{0}, \varepsilon_{1}, \varepsilon_{2}, \ldots$ be an arbitrary infinite sequence of 0 's and 1's. Since $p_{n}\left(A\left(\varepsilon_{0}, \varepsilon_{1}, \ldots, \varepsilon_{n}\right)\right)=I\left(\varepsilon_{0}, \varepsilon_{1}, \ldots, \varepsilon_{n}\right)$ and $f_{n}$ restricted to $I\left(\varepsilon_{0}, \varepsilon_{1}, \ldots, \varepsilon_{n}\right)$ is a homeomorphism onto $I\left(\varepsilon_{0}, \varepsilon_{1}, \ldots, \varepsilon_{n+1}\right)$, we have $\bigcap_{n=0}^{\infty} A\left(\varepsilon_{0}, \varepsilon_{1}, \ldots, \varepsilon_{n}\right)$ is an arc. Since $A\left(\varepsilon_{0}, \varepsilon_{1}, \ldots, \varepsilon_{n}\right)$ is a generalized end layer, we have that $\bigcap_{n=0}^{\infty} A\left(\varepsilon_{0}, \varepsilon_{1}, \ldots, \varepsilon_{n}\right)$ is a generalized end layer. So $X$ has uncountably many nondegenerate generalized end layers. 


\section{4. $\varepsilon$-MAPS FROM HEREDITARILY DECOMPOSABLE CHAINABLE CONTINUA}

The following lemma is a restatement of Lemma 7, p. 388 in [3] and the proof will be omitted.

Lemma 4.1. Suppose $C$ is an hereditarily decomposable chainable continuum and $\eta>0$. There is an $\eta$-map $h: C \rightarrow[0,1]$ so that $h(l(C))=0$ and $h(r(C))=1$.

Lemma 4.2. Suppose $C$ is a chainable, hereditarily decomposable continuum, $A$ and $B$ are end layers of $C, \varepsilon>0, f$ is an $\varepsilon$-map from $A \cup B$ into $\mathbb{R}$ (the reals) with $f(A)=\left[a_{1}, a_{2}\right], f(B)=\left[b_{1}, b_{2}\right]$ and $a_{2}<b_{1}$. Let $C_{A}$ and $C_{B}$ be two disjoint subcontinua of $C$ so that $A \subset \operatorname{int}\left(C_{A}\right)$ and $B \subset \operatorname{int}\left(C_{B}\right)$. Let $\mathscr{H}$ be a finite collection of points or intervals contained in $\left[a_{1}, a_{2}\right] \cup\left[b_{1}, b_{2}\right]$. If $\delta>0$, there is an $\varepsilon$-map $\bar{f}: C \rightarrow\left[a_{1}, b_{2}\right]$ with $\bar{f} \mid A \cup B=f, \bar{f}^{-1}\left(\left[a_{1}, a_{2}\right]\right) \subset \operatorname{int}\left(C_{A}\right)$, $\bar{f}^{-1}\left(\left[b_{1}, b_{2}\right]\right) \subset \operatorname{int}\left(C_{B}\right)$, and $\bar{f}^{-1}(I) \subset \mathscr{B}\left(f^{-1}(I), \delta\right)$ for each $I \in \mathscr{H}$.

Proof of Lemma 4.2. Take $a \in A$ and $b \in B$ so that $f(a)=a_{2}$ and $f(b)=b_{1}$. Let us extend $f$ to a function sending some open set containing $A \cup B$ into $\left[a_{1}, a_{2}\right] \cup\left[b_{1}, b_{2}\right]$. Let $f_{*}$ denote the restriction of this function to a closed neighborhood $U$ of $A \cup B$ so that $f_{*}$ is an $\varepsilon$-map and $f_{*}^{-1}(t) \subset \mathscr{B}\left(f^{-1}(t), \delta\right)$ for each $t \in\left[a_{1}, a_{2}\right] \cup\left[b_{1}, b_{2}\right]$. We can assume that $U \subset \operatorname{int}\left(C_{A}\right) \cap \operatorname{int}\left(C_{B}\right)$.

Let $m_{a}$ be a number so that $m_{a}<a_{2}, \operatorname{diam}\left(f_{*}^{-1}\left(\left[m_{a}, a_{2}\right]\right)\right)<\varepsilon$, and for each $I \in \mathscr{H}$ if $I \cap\left[m_{a}, a_{2}\right] \neq \varnothing$ then $a_{2} \in I$. Similarly, let $m_{b}$ be a number so that $m_{b}>a_{2}, \operatorname{diam}\left(f_{*}^{-1}\left(\left[b_{1}, m_{b}\right]\right)\right)<\varepsilon$, and for each $I \in \mathscr{H}$ if $I \cap\left[b_{1}, m_{b}\right] \neq \varnothing$ then $b_{1} \in I$.

Let $g: C \rightarrow[0,1]$ be a Kuratowski function with $g(A)=0$ and $g(B)=1$. Let $t_{a}$ and $t_{b}$ be so that $C \backslash g^{-1}\left(\left(t_{a}, t_{b}\right)\right) \subset U$.

Let $\eta$ be a positive number so that

(1) $\eta<\varepsilon$

(2) $\eta<\delta$

(3) $\eta<\varepsilon-\operatorname{diam}\left(f_{*}^{-1}\left(\left[m_{a}, a_{2}\right]\right)\right)$,

(4) $\eta<\varepsilon-\operatorname{diam}\left(f_{*}^{-1}\left(\left[b_{1}, m_{b}\right]\right)\right)$,

(5) $\mathscr{B}(a, \eta) \subset f_{*}^{-1}\left(\left[m_{a}, a_{2}\right]\right)$,

(6) $\mathscr{B}(b, \eta) \subset f_{*}^{-1}\left(\left[b_{1}, m_{b}\right]\right)$,

(7) $\mathscr{B}(A, \eta) \cap g^{-1}\left(\left[t_{a}, 1\right]\right)=\varnothing$ and

(8) $\mathscr{B}(B, \eta) \cap g^{-1}\left(\left[0, t_{b}\right]\right)=\varnothing$.

Let $h$ be an $\eta$-map from $C$ to $\mathbb{R}$ so that $h(a)=a_{2}$ and $h(b)=b_{1}$.

Let $c_{a}, c_{b}, d_{a}, d_{b}$ be such that $a_{2}<d_{a}<c_{a}<c_{b}<d_{b}<b_{1}$,

$$
\operatorname{diam}\left(h^{-1}\left(\left[a_{2}, c_{a}\right]\right)\right)<\eta \text { and } \operatorname{diam}\left(h^{-1}\left(\left[c_{b}, b_{1}\right]\right)\right)<\eta \text {. }
$$

Since $g^{-1}\left(\left[t_{a}, 1\right]\right)$ is connected, $\mathscr{B}(a, \eta) \cap g^{-1}\left(\left[t_{a}, 1\right]\right)=\varnothing, h^{-1}\left(\left[a_{2}, d_{a}\right]\right)$ $\subset \mathscr{B}(a, \eta)$ and $b_{1} \in h\left(g^{-1}\left(\left[t_{a}, 1\right]\right)\right)$, we have that $g^{-1}\left(\left[t_{a}, 1\right]\right) \subset h^{-1}\left(\left[d_{a}, \infty\right)\right)$. Similarly we can prove that $g^{-1}\left(\left[0, t_{b}\right]\right) \subset h^{-1}\left(\left(-\infty, d_{b}\right]\right)$. It follows that $g^{-1}\left(\left[t_{a}, t_{b}\right]\right) \subset h^{-1}\left(\left[d_{a}, d_{b}\right]\right)$. Let $S$ be the component of $h^{-1}\left(\left[d_{a}, d_{b}\right]\right)$ containing $g^{-1}\left(\left[t_{a}, t_{b}\right]\right)$. Since $A$ and $B$ are end layers of $C, A \not \subset S, S \not \subset A$, $B \not \subset S$ and $S \not \subset B$, we have that $S \cap(A \cup B)=\varnothing$. Let $F$ be a closed and open subset of $h^{-1}\left(\left[d_{a}, d_{b}\right]\right)$ containing $S$ and missing $A \cup B$. Let $\tau$ be a continuous function from $C$ onto $[0,1]$ so that $\tau\left(h^{-1}\left(\left[c_{a}, c_{b}\right]\right)\right)=1$ and 
$\tau\left(C \backslash h^{-1}\left(\left(d_{a}, d_{b}\right)\right)\right)=0$. Our function $\bar{f}$ is defined by

$$
\bar{f}(x)=\left\{\begin{array}{l}
h(x) \quad \text { if } x \in F \cap h^{-1}\left(\left[c_{a}, a_{b}\right]\right), \\
\tau(x) h(x)+(1-\tau(x)) f_{*}(x) \quad \text { if } x \in F \backslash h^{-1}\left(\left[c_{a}, c_{b}\right]\right), \\
f_{*}(x) \text { if } x \notin F .
\end{array}\right.
$$

Observe that $\bar{f}$ is continuous.

Since $F \backslash h^{-1}\left(\left[c_{a}, c_{b}\right]\right) \subset h^{-1}\left(\left[a_{2}, c_{a}\right]\right) \cup h^{-1}\left(\left[c_{b}, b_{1}\right]\right) \subset \mathscr{B}(a, \eta) \cup \mathscr{B}(b, \eta)$, $\mathscr{B}(a, \eta) \subset f_{*}^{-1}\left(\left[m_{a}, a_{2}\right]\right), \mathscr{B}(b, \eta) \subset f_{*}^{-1}\left(\left[b_{1}, m_{b}\right]\right)$ and $h(F) \subset\left[d_{a}, d_{b}\right]$ we have that $\bar{f}(F) \subset\left[m_{a}, m_{b}\right]$.

To prove that $\operatorname{diam}\left(\bar{f}^{-1}(t)\right)<\varepsilon$, we will consider the cases where (i) $t<m_{a}$ or $m_{b}<t$, (ii) $m_{a} \leq t \leq c_{a}$, (iii) $c_{b} \leq t \leq m_{b}$ and (iv) $c_{a}<t<c_{b}$.

In case (i) we have that $\bar{f}^{-1}(t) \cap F=\varnothing$ and thus $\bar{f}^{-1}(t)=f_{*}^{-1}(t)$ and the proof follows from the fact that $f_{*}$ is an $\varepsilon$-map.

In case (ii) we have that $\bar{f}^{-1}(t) \subset f_{*}^{-1}\left(\left[m_{a}, a_{2}\right]\right) \cup h^{-1}\left(\left[a_{2}, c_{a}\right]\right)$. Since $\eta<$ $\varepsilon-\operatorname{diam}\left(f_{*}^{-1}\left(\left[m_{a}, a_{2}\right]\right)\right), \operatorname{diam}\left(h^{-1}\left(\left[a_{2}, c_{a}\right]\right)\right)<\eta$ and $a \in f_{*}^{-1}\left(\left[m_{a}, a_{2}\right]\right) \cap$ $h^{-1}\left(\left[a_{2}, c_{a}\right]\right)$, we have that $\operatorname{diam}\left(\bar{f}^{-1}(t)\right)<\varepsilon$.

The proof in case (iii) is analogous to that in case (ii).

In case (iv) we have that $\bar{f}^{-1}(t) \subset h^{-1}(t)$ and the proof follows from the fact that $\eta<\varepsilon$ and $h$ is an $\eta$-map.

To complete the proof of the lemma we have to show that $\bar{f}^{-1}(I) \subset$ $\mathscr{B}\left(f^{-1}(I), \delta\right)$ for each $I \in \mathscr{H}$. If $I \subset\left[a_{1}, m_{a}\right)$ then $\bar{f}^{-1}(I)=f_{*}^{-1}(I)$ and since $f_{*}^{-1}(t) \subset \mathscr{B}\left(f^{-1}(t), \delta\right)$ for each $t \in\left[a_{1}, a_{2}\right] \cup\left[b_{1}, b_{2}\right]$, we have that $\bar{f}^{-1}(I) \subset \mathscr{B}\left(f^{-1}(I), \delta\right)$.

If $I \cap\left[m_{a}, a_{2}\right] \neq \varnothing$ then $a_{2} \in I$. In this case $\bar{f}^{-1}(I) \subset f_{*}^{-1}(I) \cup h^{-1}\left(\left[a_{2}, c_{a}\right]\right)$. Since $f_{*}^{-1}(I) \subset \mathscr{B}\left(f^{-1}(I), \delta\right), \operatorname{diam}\left(h^{-1}\left(\left[a_{2}, c_{a}\right]\right)\right)<\eta<\delta$ and $a \in f^{-1}(I) \cap$ $h^{-1}\left(\left[a_{2}, c_{a}\right]\right)$, we have that $\bar{f}^{-1}(I) \subset \mathscr{B}\left(f^{-1}(I), \delta\right)$.

The proof in case $I \subset\left[b_{1}, b_{2}\right]$ is similar.

If $\mathscr{K}$ is a collection of sets, the union of all elements of $\mathscr{K}$ will be denoted by $\mathscr{K}^{*}$.

Lemma 4.3. Suppose $C$ is an hereditarily decomposable chainable continuum, $\mathscr{A}=\left\{L_{1}, L_{2}, \ldots, L_{n}\right\}$ is a collection of layers of $C$ listed in order, in the sense that if $1 \leq i<j \leq n, x \in L_{i}$ and $y \in L_{j}$ then $x<y$. Let $D_{1}, D_{2}, \ldots, D_{n}$ be a collection of mutually disjoint subcontinua of $C$ so that $L_{i} \subset \operatorname{int}\left(D_{i}\right)$ for each $i=1, \ldots, n$. Suppose also that $\varepsilon>0$ and $a_{1} \leq b_{1}<a_{2} \leq b_{2}<\cdots<a_{n} \leq b_{n}$ are real numbers. Let $f: \mathscr{A}^{*} \rightarrow \mathbb{R}$ be an $\varepsilon$-map such that $f\left(L_{i}\right)=\left[a_{i}, b_{i}\right]$ where

$$
a_{i} \in f\left(\left[l(C), l\left(L_{i}\right)\right] \cap L_{i}\right) \quad \text { and } \quad b_{i} \in f\left(\left[r\left(L_{i}\right), r(C)\right] \cap L_{i}\right)
$$

for each $i=1, \ldots, n$. Then there is an $\varepsilon$-map $\bar{f}: C \rightarrow \mathbb{R}$ so that $\bar{f}$ extends $f, \bar{f}(l(C)) \leq \bar{f}(x) \leq \bar{f}(r(C))$ for each $x \in C$, and $\bar{f}^{-1}\left(\left[a_{i}, b_{i}\right]\right) \subset D_{i}$, $\bar{f}\left(\left[l(C), r\left(L_{i}\right)\right]\right)=\left[\bar{f}(l(C)), b_{i}\right]$ and $\bar{f}\left(\left[l\left(L_{i}\right), r(C)\right]\right)=\left[a_{i}, \bar{f}(r(C))\right]$ for $i=$ $1, \ldots, n$.

Proof of Lemma 4.3. Observe that $f(l(C))=a_{1}$ if $l(C) \in L_{1}$, and $f(r(C))=b_{n}$ if $r(C) \in L_{n}$. Without loss of generality we may assume that $L_{1}$ and $L_{n}$ 
are the end layers of $C$. (If it is needed, we may simply add a layer to the collection $\mathscr{A}$ and extend $f$ applying Lemma 4.1.) For each $i=1, \ldots, n-1$ let $C_{i}=\left[r\left(L_{i}\right), l\left(L_{i+1}\right)\right], A_{i}=C_{i} \cap L_{i}, B_{i}=C_{i} \cap L_{i+1}, c_{i}=\min f\left(A_{i}\right)$ and $d_{i}=\max f\left(B_{i}\right)$. Observe that $L_{i+1}=B_{i} \cup A_{i+1}$ for $i=1, \ldots,(n-2)$, and consequently $f\left(B_{i}\right) \cup f\left(A_{i+1}\right)=\left[c_{i+1}, d_{i}\right]$ with $c_{i+1} \leq d_{i}$. Since $f$ is an $\varepsilon$-map there is a positive number $\delta$ so that $f$ is an $(\varepsilon-2 \delta)$-map. There is a finite collection $\mathscr{H}$ of points and intervals contained in $\mathscr{A}^{*}$ so that $\mathscr{H}^{*}=f\left(\mathscr{A}^{*}\right)$, $\operatorname{diam}\left(f^{-1}(I)\right)<\varepsilon-2 \delta$ for each $I \in \mathscr{H}$, and for each $t \in\left[c_{i+1}, d_{i}\right]$ where $i=$ $1, \ldots, n-2$ there is $I \in \mathscr{H}$ so that $t \in I \subset\left[c_{i+1}, d_{i}\right]$. Let $\mathscr{H}_{i}=\{I \in \mathscr{H} \mid I \subset$ $\left.f\left(A_{i} \cup B_{i}\right)\right\}$. Applying Lemma 4.2 for each of the continua $C_{i}$ with end layers $A_{i}$ and $B_{i}$ we get an $\varepsilon$-map $f_{i}: C_{i} \rightarrow\left[c_{i}, d_{i}\right]$ so that $f_{i}\left|A_{i} \cup B_{i}=f\right| A_{i} \cup B_{i}$, $f_{i}^{-1}\left(f_{i}\left(A_{i}\right)\right) \subset D_{i}, f_{i}^{-1}\left(f_{i}\left(B_{i}\right)\right) \subset D_{i+1}$ and $f_{i}^{-1}(I) \subset \mathscr{B}\left(f^{-1}(I), \delta\right)$ for each $I \in \mathscr{H}_{i}$. Let $\bar{f}=\bigcup_{i=0}^{n-1} f_{i}$. We will prove that $\bar{f}$ is an $\varepsilon$-map, the other required properties of $\bar{f}$ are apparent.

Take $t \in\left[a_{1}, b_{n}\right]$. Let $i$ be the smallest integer so that $t \in\left[c_{i}, d_{i}\right]$. If $i=n-1$ or $t<c_{i+1}$ then $\bar{f}^{-1}(t)=f_{i}^{-1}(t)$, so we can assume that $t \in$ $\left[c_{i+1}, d_{i}\right]$. There is $I \in \mathscr{H}_{i} \cap \mathscr{H}_{i+1}$ so that $t \in I$. Now, $\bar{f}^{-1}(t)=f_{i}^{-1}(t) \cup$ $f_{i+1}^{-1}(t) \subset f_{i}^{-1}(I) \cup f_{i+1}^{-1}(I) \subset \mathscr{B}\left(f^{-1}(I), \delta\right)$. Since $\operatorname{diam}\left(f^{-1}(I)\right)<\varepsilon-2 \delta$, $\operatorname{diam}\left(\mathscr{B}\left(f^{-1}(I), \delta\right)\right)<\varepsilon$ and consequently $\operatorname{diam}\left(\bar{f}^{-1}(t)\right)<\varepsilon$.

Definition. Let $\mathscr{K}$ be a finite collection of subcontinua of an hereditarily decomposable chainable continuum $X$.

Suppose that $\mathscr{K}$ is semimonotone in the sense that if $A, B \in \mathscr{K}$ then either $A \cap B=\varnothing$, or $A \subset B$ or $B \subset A$. We will denote by $E(\mathscr{K})$ the set $\{x \mid x=l(C)$ or $x=r(C)$ for some $C \in \mathscr{K}\}$. If $C \in \mathscr{K}$ let $\mathscr{K}(C)$ be the collection of $K \in \mathscr{K}$ so that $K \subset C, K \neq C$ and $K$ is contained in no other element of $\mathscr{K}$ which is contained in $C$.

Let $f$ be a map of $\mathscr{K}^{*}$ into an ordered arc. We will say that $f$ respects the order of $\mathscr{K}$ if

(i) if $a \in E(\mathscr{K}), b \in E(\mathscr{K})$ and $a<b$ then $f(a)<f(b)$, and

(ii) $f(C)=[f(l(C)), f(r(C))]$ for every $C \in \mathscr{K}$.

In the case where the domain of $f$ is an interval we will say that $f$ strictly respects the order of $\mathscr{K}$ if the above definition is satisfied with condition (ii) replaced by

(ii) $f(l(C), r(C))=(f(l(C)), f(r(C)))$ for every $C \in \mathscr{K}$.

We will say that $f$ is controlled by $\mathscr{K}$ if

(i) $f$ respects the order of $\mathscr{K}$,

(ii) if $C \in \mathscr{K}$ and $D \in \mathscr{K}(C)$ then either

$$
\begin{gathered}
f([l(C), r(D)])=[f(l(C)), f(r(D))] \text { or } \\
f([l(D), r(C)])=[f(l(D)), f(r(C))], \text { and }
\end{gathered}
$$

(iii) if $C \in \mathscr{K}$ and $\mathscr{K}(C)$ contains at least two elements then for each $D \in \mathscr{K}(C)(*)$ holds and there is a continuum $C(D) \subset C$ so that $f^{-1}(f(D)) \cap$ $C \subset \operatorname{int}_{C}(C(D))$ and $C\left(D_{1}\right) \cap C\left(D_{2}\right)=\varnothing$ for every two different $D_{1}$ and $D_{2}$ from $K(C)$. 
We will say that $f$ is strictly controlled by $\mathscr{K}$ (again, this will be used only when the domain of $f$ is an interval) if $f$ strictly respects the order of $\mathscr{K}$ and the above definition is satisfied with the conditions $(*)$ and $(* *)$ replaced by the following

$(*)_{S}$

$(* *)_{S}$

$$
\begin{aligned}
& f([l(C), r(D)))=[f(l(C)), f(r(D))), \\
& f((l(D), r(C)])=(f(l(D)), f(r(C))] .
\end{aligned}
$$

If $\mathscr{K}$ is a finite collection of generalized layers, we say that $\mathscr{K}$ is complete if $X \in \mathscr{K}$ and

(i) if $C \in \mathscr{K}$ and $\mathscr{K}(C)$ contains at least two elements then all elements of $\mathscr{K}(C)$ are layers of $C$.

(ii) if $A \in \mathscr{K}$ and $B \in \mathscr{K}$ then there is $C \in \mathscr{K}$ so that $A \cup B \subset C$.

Lemma 4.4. Let $\mathscr{Z}$ be a finite collection of generalized layers of an hereditarily decomposable chainable continuum $X$. Then for each positive number $\varepsilon$ there is a complete finite collection $\mathscr{K}$ of generalized layers of $X$ and there is an E-map $f: X \rightarrow \mathbb{R}$ so that $\mathscr{Z} \subset \mathscr{K}$ and $f$ is controlled by $\mathscr{K}$.

Proof of Lemma 4.4. Since each finite collection $\mathscr{Z}$ of generalized layers can be extended to a finite, complete collection, we may assume that $\mathscr{Z}$ is complete. By adding to the collection $\{l(M)\}$ for each minimal (under inclusion) element $M$ of $\mathscr{Z}$, we can assume that each minimal element of $\mathscr{Z}$ is a single point. We define $f$ on the union of one point elements of $\mathscr{Z}$, and then extend it gradually to other elements of $\mathscr{Z}$. Suppose that $C \in \mathscr{Z}$ and $f_{0}$ is an $\varepsilon$-map defined on $(\mathscr{Z}(C))^{*}$ so that $f_{0}$ is controlled by some collection $\mathscr{K}_{0}$ of generalized layers of $X$. To complete the proof it is enough to extend $f_{0}$ to an $\varepsilon$-map defined on $C$ and controlled by some complete collection $\mathscr{K}$ containing $\mathscr{K}_{0}$.

Case: $\mathscr{Z}(C)$ has more than one element. Let $\mathscr{Z}(C)=\left\{L_{1}, L_{2}, \ldots, L_{n}\right\}$. Since $\mathscr{Z}$ is complete the elements of $\mathscr{Z}(C)$ are layers of $C$. There is a collection $D_{1}, D_{2}, \ldots, D_{n}$ of mutually disjoint subcontinua of $C$ so that $L_{i} \subset$ int $_{C}\left(D_{i}\right)$ for each $i=1, \ldots, n$. Applying Lemma 4.3, we can extend $f_{0}$ to C.

Case: $\mathscr{Z}(C)$ has one element. Let $C_{0}$ be the element of $\mathscr{Z}(C)$. We shall construct a sequence $C_{0} \subset C_{1} \subset C_{2} \cdots$ of generalized layers of $L$, and a sequence of functions $f_{0} \subset f_{1} \subset f_{2} \cdots$ so $f_{i}: C_{i} \rightarrow \mathbb{R}$ is an $\varepsilon$-map which respects the order of $\left\{C_{i}\right\}$. Moreover, for each $i$ we will have that either

$(*)_{i}$

$$
\begin{gathered}
f_{i+1}\left(\left[l\left(C_{i+1}\right), r\left(C_{i}\right)\right]\right)=\left[f_{i+1}\left(l\left(C_{i+1}\right)\right), f_{i}\left(r\left(C_{i}\right)\right)\right], \text { or } \\
f_{i+1}\left(\left[l\left(C_{i}\right), r\left(C_{i+1}\right)\right]\right)=\left[f_{i}\left(l\left(C_{i}\right)\right), f_{i+1}\left(r\left(C_{i+1}\right)\right)\right], \text { and }
\end{gathered}
$$

if $C_{i}$ is a layer of $C_{i+1}$ the condition $(*)_{i}$ will hold.

Since any chain of generalized layers is well ordered, this process will necessarily end with some $C_{k}=C$. So, to complete the proof in the case considered it suffices to take one step of the construction.

Suppose $C_{i} \neq C$ and $f_{i}$ are given with $f\left(l\left(C_{i}\right)\right)=a$ and $f\left(r\left(C_{i}\right)\right)=b$. Let $f_{*}: X \rightarrow[a, b]$ be a continuous extension of $f_{i}$. Let $U$ be an open set (in $C$ ) containing $C_{i}$ so that $f_{*} \mid U$ is an $\varepsilon$-map. There is a continuum $C_{i+1}$ which is either $C$ or a generalized layer of $C$ such that a layer $Y$ of $C_{i+1}$ contains 
$C_{i}$ and is contained in $U$. Let $L=\left[l\left(C_{i+1}\right), l(Y)\right]$ and $R=\left[r(Y), r\left(C_{i+1}\right)\right]$. Observe that $C_{i+1}=L \cup R$.

If $l\left(C_{i}\right) \in L$ and $r\left(C_{i}\right) \in R$, then $a \in f_{*}(L \cap Y), b \in f_{*}(R \cap Y)$ and Lemma 4.3 can be applied to extend $f_{*} \mid Y$ to an $\varepsilon$-map $f_{i+1}: C_{i+1} \rightarrow \mathbb{R}$ such that $f_{i+1}(L)=\left[f_{i+1}\left(l\left(C_{i+1}\right)\right), b\right]$ and $f_{i+1}(R)=\left[a, f_{i+1}\left(r\left(C_{i+1}\right)\right)\right]$, and in this case the construction is completed. So, without loss of generality, we may assume that $r\left(C_{i}\right) \notin R$ and consequently $r\left(C_{i}\right) \in L$. Note that in this case $C_{i} \neq Y$, so $C_{i}$ is not a layer of $C_{i+1}$. Since $l\left(C_{i+1}\right) \leq l\left(C_{i}\right) \leq r\left(C_{i}\right)$, we have that $l\left(C_{i}\right) \in L$.

Let $S=\left[r\left(C_{i}\right), r(Y)\right]$, and let $A$ and $B$ be the layers of $S$ containing $r\left(C_{i}\right)$ and $r(Y)$ respectively. Let $c=\min f_{*}(A)$. It follows from Lemma 4.1 that there is an $\varepsilon$-map $h$ of $B$ onto an interval $[d, e]$ with $d>b$ and $h(r(Y))=e$. Let $h_{*}: A \cup B \rightarrow \mathbb{R}$ be so that $h_{*}\left|A=f_{*}\right| A$ and $h_{*} \mid A=h$. There is a positive number $\delta$ so that $f_{*} \mid Y$ is an $(\varepsilon-2 \delta)$-map. There is a finite collection $\mathscr{H}$ of points and intervals contained in $[c, b]$ so that $\mathscr{H}^{*}=[c, b], \operatorname{diam}\left(f_{*}^{-1}(I)\right)<$ $\varepsilon-2 \delta$ for each $I \in \mathscr{H}$. Applying Lemma 4.2 we get an $\varepsilon$-map $\bar{h}: S \rightarrow[c, e]$ so that $\bar{h}\left|A \cup B=h_{*}\right| A \cup B$ and $\bar{h}^{-1}(I) \subset \mathscr{B}\left(f_{*}^{-1}(I), \delta\right)$ for each $I \in \mathscr{H}$. Let $g: Y \rightarrow[a, e]$ be defined by $g(y)=\bar{h}(y)$ if $y \in S$ and $g(y)=f_{*}(y)$ otherwise. Observe that $g$ is an $\varepsilon$-map so that $a \in g\left(\left[l\left(C_{i+1}\right), l(Y)\right] \cap Y\right)=g(L \cap Y)$ and $e \in g\left(\left[r\left(C_{i+1}\right), r(Y)\right] \cap Y\right)$. We then apply Lemma 4.3 to extend $g$ to an $\varepsilon$ map $f_{i+1}: C_{i+1} \rightarrow \mathbb{R}$ so that $f_{i+1}\left(l\left(C_{i+1}\right)\right) \leq f_{i+1}(x) \leq f_{i+1}\left(r\left(C_{i+1}\right)\right)$ for each $x \in C_{i+1}$, and $\left.f_{i+1}\left(\left[l(Y), r\left(C_{i+1}\right)\right)\right]\right)=\left[a, f_{i+1}\left(r\left(C_{i+1}\right)\right)\right]$. Observe that $(* *)_{i}$ is satisfied and the construction is completed.

Lemma 4.5. Let $\mathscr{K}$ be a complete finite collection of generalized layers of an hereditarily decomposable chainable continuum $X$. Suppose that $f: X \rightarrow[0,1]$ is a function controlled by $\mathscr{K}$ and $\varepsilon$ is a positive number. Then there is a positive number $\delta$ so that for each $\delta$-map $g: X \rightarrow[0,1]$ which respects the order of $\mathscr{K}$ there is a map $p:[0,1] \rightarrow[0,1]$ such that

(i) $p$ is strictly controlled by $g(\mathscr{K})$,

(ii) $p(g(e))=f(e)$ for each $e \in E(\mathscr{K})$, and

(iii) $|p(g(x))-f(x)|<\varepsilon$ for each $x \in X$.

Proof of Lemma 4.5. For each $C \in \mathscr{K}$ for which $\mathscr{K}(C)$ contains more than one element and for each $D \in \mathscr{K}(C)$ let $C(D) \subset C$ be a continuum so that $f^{-1}(f(D)) \cap C \subset \operatorname{int}_{C}(C(D))$ and $C\left(D_{1}\right) \cap C\left(D_{2}\right)=\varnothing$ for every two different $D_{1}$ and $D_{2}$ from $\mathscr{K}(C)$. There is a positive number $\alpha$ so that for each $C \in \mathscr{K}$ the distance between $C\left(D_{1}\right)$ and $C\left(D_{2}\right)$ is greater than $\alpha$ for every two different $D_{1}$ and $D_{2}$ from $\mathscr{K}(C)$. There is a positive number $\xi$ so that $f^{-1}(\mathscr{B}(f(D), \xi)) \cap C \subset \operatorname{int}_{C}(C(D))$. There is a positive number $\eta$ such that $\eta<\varepsilon / 2, \eta<\xi / 5$ and $\left|f\left(e_{1}\right)-f\left(e_{2}\right)\right|>2 \eta$ for each two different points $e_{1}$ and $e_{2}$ from $E(\mathscr{K})$. Let $\delta<\alpha$ be a positive number so that for each $x, y \in X$ if $|x-y|<\delta$ then $|f(x)-f(y)|<\eta$.

Now suppose $g: X \rightarrow[0,1]$ is a $\delta$-map which respects the order of $\mathscr{K}$. Let $T$ to be a finite subset of $[0,1]$ such that $g(E(\mathscr{K})) \subset T$ and $\operatorname{diam}\left(g^{-1}(A)\right)<$ $\delta$ for each component $A$ of $[0,1] \backslash T$. For each $t \in T$ let $q(t)$ be a point of $g^{-1}(t)$ chosen from the smallest element of $\mathscr{K}$ possible so that $q(g(e))=e$ for each $e \in E(\mathscr{K})$. For each $t \in T \backslash g(E(\mathscr{K}))$ let $(a(t), b(t))$ be the component of $[0,1] \backslash g(E(\mathscr{K}))$ containing $t$. Observe that $f(q(a(t)))<f(q(b(t)))$. Let 
$\tau: T \rightarrow\{-\eta, 0, \eta\}$ be defined by

$$
\tau(t)= \begin{cases}\eta & \text { if } t \in T \backslash g(E(\mathscr{K})) \text { and } f(q(t)) \leq f(q(a(t))), \\ -\eta & \text { if } t \in T \backslash g(E(\mathscr{K})) \text { and } f(q(t)) \geq f(q(b(t))), \\ 0 & \text { otherwise. }\end{cases}
$$

Define $p(t)=f(q(t))+\tau(t)$ for $t \in T$ and then extend $p$ to $[0,1]$ by making it linear on each component of $[0,1] \backslash T$.

It is clear from the construction that (ii) is satisfied. To see that (iii) is satisfied, take $x \in X$. Let $t_{1}$ and $t_{2}$ be consecutive elements of $T$ so that $t_{1} \leq g(x) \leq t_{2}$. We have that $\operatorname{diam}\left(g^{-1}\left(\left[t_{1}, t_{2}\right]\right)\right)<\delta$ and thus $\left|q\left(t_{i}\right)-x\right|<\delta$ for $i=1,2$. So $\left|f\left(q\left(t_{i}\right)\right)-f(x)\right|<\eta$. We also know that $\left|p\left(t_{i}\right)-f\left(q\left(t_{i}\right)\right)\right| \leq \eta$. So $\left|f(x)-p\left(t_{i}\right)\right|<2 \eta$ and consequently $|f(x)-p(g(x))|<2 \eta<\varepsilon$.

To see that $p$ is strictly controlled by $g(\mathscr{K})$ observe first that the monotonicity of $p$ on $E(g(\mathscr{K}))$ is a direct consequence of the construction. Now let $C \in \mathscr{K}$. Let $c_{0}=g(l(C))$ and $c_{1}=g(r(C))$. To see that $p$ strictly respects the order of $g(\mathscr{K})$ it suffices to show that if $t \in T$ and $c_{0}<t<c_{1}$ then $p\left(c_{0}\right)<p(t)<p\left(c_{1}\right)$.

Observe that by the choice of $q(t)$ we have that $q(t) \in C$. We also have that $p\left(c_{0}\right) \leq p(a(t))<p(b(t))<p\left(c_{1}\right)$. We consider three cases: (1) $f(q(t)) \leq$ $p(a(t)),(2) p(a(t))<f(q(t))<p(b(t))$ and (3) $p(b(t)) \leq f(q(t))$. Observe that case 2 is obvious. In case 1 we have $f(q(t)) \geq p\left(c_{0}\right)=f(l(C))$ and $p(t)=f(q(t))+\eta>p\left(c_{0}\right)$, but, since $p(b(t))-p(a(t))>\eta$ we have that $p(t)<p(b(t)) \leq p\left(c_{1}\right)$. The proof in case 3 is just like that in case 1 .

Now we will prove that if $f$ satisfies condition $(*)$ of the definition of being controlled by $\mathscr{K}$ then $p$ satisfies $(*)_{s}$ with respect to $g(\mathscr{K})$. To that end it suffices to prove that if $C \in \mathscr{K}, D \in \mathscr{K}(C), f([l(C), r(D)])=[f(l(C)), f(r(D))]$ and $t \in T \cap[g(l(C)), g(r(D)))$ then $f(l(C)) \leq p(t)<f(r(D))$.

We may assume that $t \notin E(g(\mathscr{K}))=g(E(\mathscr{K}))$. So we have $g(l(C)) \leq$ $a(t)<t<b(t) \leq g(r(D))$. Let $z \in[l(C), r(D)]$ so that $g(z)=t$. Since $g$ is a $\delta$-map we have that $|f(z)-f(q(t))|<\eta$. We will consider three cases: (a) $\tau(t)=0$, (b) $\tau(t)=\eta$ and (c) $\tau(t)=-\eta$. In case (a) we have $f(q(a(t)))<$ $f(q(t))<f(q(b(t)))$ so $f(l(C)) \leq f(q(a(t)))<f(q(t))<f(q(b(t))) \leq f(r(D))$. In case (b) we have $f(q(t) \leq f(q(a(t)))$ so $p(t)=f(q(t))+\eta \leq f(q(a(t)))+\eta<$ $f(q(b(t))) \leq f(r(D))$ and $f(l(C)) \leq f(z)<f(q(t))+\eta=p(t)$. Case (c) is a mirror image of case (b).

So if $f$ satisfies condition $(*)$ of the definition of being controlled by $\mathscr{K}$ then $p$ satisfies $(*)_{s}$ with respect to $g(\mathscr{K})$. The proof in case $(* *)$ for $f$ implying $(* *)_{s}$ is again a mirror image of the one presented and will be omitted.

To complete the proof of the lemma it remains to show that $p$ satisfies condition (iii) of the definition of being controlled $g(\mathscr{K})$. Let $C \in \mathscr{K}$ be so that $\mathscr{K}(C)$ contains more than one element and let $D \in \mathscr{K}(C)$. We must exhibit continua (intervals) which we denote by $G(D)$ so that $G(D) \subset g(C)$, $p^{-1}(p(g(D))) \cap g(C) \subset \operatorname{int}_{g(C)}(G(D))$ and $G\left(D_{1}\right) \cap G\left(D_{2}\right)=\varnothing$ if $D_{1}$ and $D_{2}$ are different elements of $\mathscr{K}(C)$. Note that since $\delta<\alpha$, we have that $g\left(C\left(D_{1}\right)\right) \cap$ $g\left(C\left(D_{2}\right)\right)=\varnothing$ if $D_{1}$ and $D_{2}$ are different elements of $\mathscr{K}(C)$. We choose $G(D)$ to be $g(C(D))$ and observe that it will suffice to get $p^{-1}(p(g(D))) \cap g(C) \subset$ $G(D)$ since these intervals could be fattened slightly and still not meet each other.

Let $v$ be an arbitrary point of $p^{-1}(p(g(D))) \cap g(C)$. Let $t_{1}$ and $t_{2}$ be in 
$T$ so that $t_{1}$ is the greatest point of $T$ which is less than or equal to $v$ and $t_{2}$ is the least point of $T$ which is greater than or equal to $v$. Since $v \in g(C)$ we have $l(g(C)) \leq t_{1} \leq t_{2} \leq r(g(D))$ so $q\left(t_{i}\right) \in C$ for $i=1,2$.

There is a point $d \in D$ so that $p(g(d))=p(v)$. We observed earlier that $|f(x)-p(g(x))|<2 \eta$ for each $x \in X$, so we have that $|f(d)-p(g(d))|<2 \eta$. Since $p$ is linear on $\left[t_{1}, t_{2}\right], p(v)$ is between $p\left(t_{1}\right)$ and $p\left(t_{2}\right)$. These numbers differ from $f\left(q\left(t_{1}\right)\right)$ and $f\left(q\left(t_{2}\right)\right)$ by at most $\eta$ in each case, and, since $\mid q\left(t_{1}\right)$ $q\left(t_{2}\right)|<\delta| f,\left(q\left(t_{1}\right)\right)-f\left(q\left(t_{2}\right)\right) \mid<\eta$, we have that the interval $\left[p\left(t_{1}\right), p\left(t_{2}\right)\right]$ has length less than $3 \eta$. Thus $\left|f\left(q\left(t_{i}\right)\right)-f(d)\right| \leq\left|f\left(q\left(t_{i}\right)\right)-p(v)\right|+|p(v)-f(d)|<$ $3 \eta+2 \eta=5 \eta$ for $i=1,2$. Since $q\left(t_{1}\right)$ and $q\left(t_{2}\right)$ belong to $C$, and $5 \eta<\xi$, we have that $q\left(t_{1}\right)$ and $q\left(t_{2}\right)$ are both in $C(D)$. So $t_{1}$ and $t_{2}$ are in $g(C(D))$ and $v$ is also, and the proof of the lemma is concluded.

\section{STRIPS IN THE PLANE}

Definition. If $S$ is a disk, $I$ is an arc contained in the boundary of $S$ and $\pi$ is a retraction of $S$ to $I$, then we will say that the triple $(S, I, \pi)$ is a strip provided that there is a homeomorphism $h$ of $S$ onto $[0,1] \times[0,1]$ so that $h\left(\pi\left(h^{-1}((x, y))\right)\right)=(0, y)$ for every $x, y \in[0,1]$. For each $s \in S$ the arc $\pi^{-1}(\pi(s))$ will be denoted by $F(\pi, s)$. Let $w(\pi)$ denote the maximum diameter of such arcs.

Suppose $(S, I, \pi)$ is a strip, $Z$ is a subset of $S$ and $\mathscr{A}$ is a semimonotone collection of subsets of $Z$. We will say that $\pi$ exposes $\mathscr{A}$ in $Z$ (or $\mathscr{A}$ is $\pi$-exposed in $Z$ ) provided that if $A \in \mathscr{A}$ and $a \in A$ then $a=\pi(a)$ or, if $C$ is the component of $F(\pi, a) \backslash\{a\}$ containing $\pi(a)$ then $C \cap Z \subset A$.

Lemma 5.1. Suppose $b, b^{\prime}, a^{\prime}$ and a are real numbers so that $b \leq b^{\prime}<$ $a^{\prime} \leq a$. Let $p:[b, a] \rightarrow[0,1]$ be a map so that $p^{-1}(p(b)) \subset\left[b, b^{\prime}\right]$ and $p^{-1}(p(a)) \subset\left[a^{\prime}, a\right]$. Let $S$ denote the square $[0,1] \times[0,1]$. Then there is $a$ map $s:[b, a] \rightarrow[0,1]$ so that $s(b)=s(a)=0, s(t)>0$ for each $t \in(b, a)$, and the map $h:[b, a] \rightarrow S$ defined by the formula $h(t)=(s(t), p(t))$ is an embedding of $[b, a]$ into $S$.

Proof of Lemma 5.1. (See Figure 1.) Without loss of generality we can assume that $b=0, a=1$ and $p(0)<p(1)$. Let $c$ be the minimum value of $p$ on the interval $\left[a^{\prime}, 1\right]$. Note that $c>p(0)$. Let $d$ be a number so that $p(0)<d<c$. Let $u$ be the last point of $[0,1]$ so that $p(u)=d$. Obviously $0<u<1$. Let $m$ be the maximum value of $p$ on the interval $[0, u]$. Observe that $m<p(1)$ and $d<p(t)$ for each $t \in(u, 1]$. Let $A$ denote the set $\{s \in S \mid s=(t, p(t))$ and $0 \leq t \leq u\}$ and let $H$ be the convex hull of $A$. For each $z \in[d, m]$ let $\psi_{0}(z)$ be the greatest real number so that $\left(\psi_{0}(z), z\right)$ is a point of $H \cap[0,1] \times\{z\}$. Observe that $\psi_{0}$ is a continuous function of $\left[m_{0}, m_{1}\right]$ and $\psi_{0}(p(u))=u$ and $\psi_{0}(p(t))<u$ for $t \in(d, m]$. There is a continuous function $\psi:[d, 1] \rightarrow[0, u]$ so that $\psi(z)=\psi_{0}(z)$ for $z \in[d, m]$ and $\psi(z)=0$ for $z \geq p(1)$. Let $\tau$ be a decreasing homeomorphism of the interval $[u, 1]$ onto the interval $[0,1]$. Define $s$ by the following formula

$$
s(t)=\left\{\begin{array}{l}
t \quad \text { if } 0 \leq t \leq u, \\
(1-\tau(t)) \psi(p(t))+u \tau(t) \quad \text { if } u \leq t \leq 1
\end{array}\right.
$$




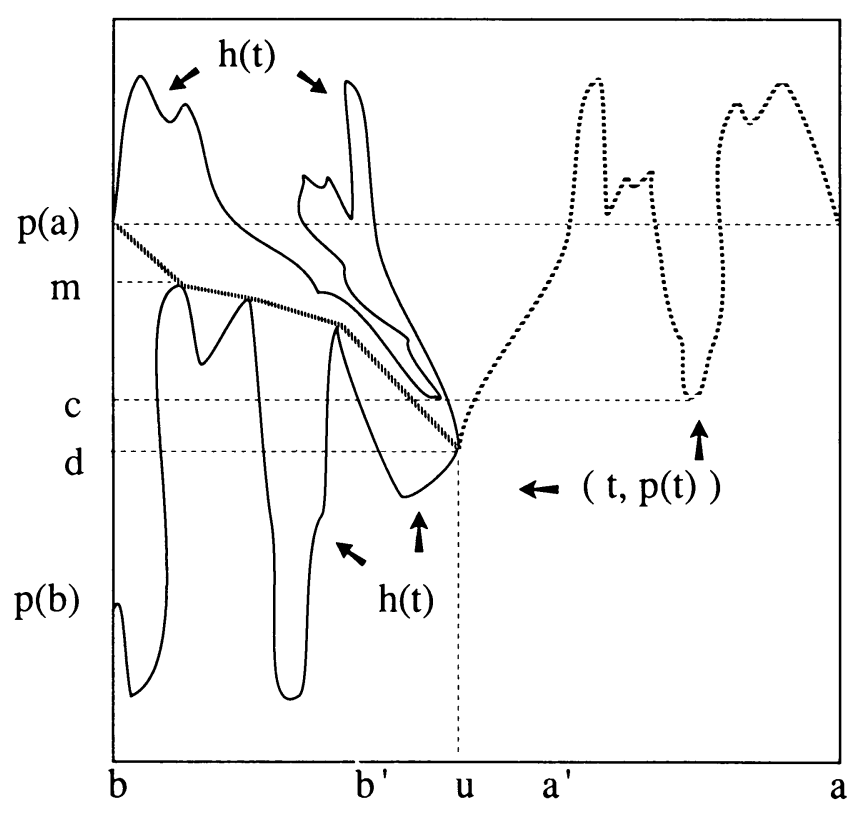

FiguRE 1

Observe that since $s(t)>\psi(p(t))$ for each $t \in(u, 1)$, we have that $h([0, u])$ $\cap h([u, 1])=\{h(u)\}$. Obviously, $h$ restricted to $[0, u]$ is an embedding, so to complete the proof of the lemma it is enough to prove that $h$ restricted to $[u, 1]$ is a bijection. Suppose $h\left(t_{0}\right)=h\left(t_{1}\right)$ for some $t_{0}, t_{1} \in[u, 1]$. Then $p\left(t_{0}\right)=p\left(t_{1}\right)$ and $\left(1-\tau\left(t_{0}\right)\right) \psi\left(p\left(t_{0}\right)\right)+u \tau\left(t_{0}\right)=\left(1-\tau\left(t_{1}\right)\right) \psi\left(p\left(t_{1}\right)\right)+u \tau\left(t_{1}\right)$. It follows that $\left(\tau\left(t_{1}\right)-\tau\left(t_{0}\right)\right)\left(u-\psi\left(p\left(t_{0}\right)\right)\right)=0$. The last equation is satisfied only if $t_{0}=t_{1}$, since $\psi(z)=u$ only if $z=p(u)=d$ and by the choice of $u$, $p(t)>d$ for each $t>u$.

Figure 2 illustrates the construction, and aspects of the proof of the following lemma.

Lemma 5.2. Suppose $\mathscr{K}$ is a finite semimonotone collection of intervals contained in $[0,1]$ with $[0,1] \in \mathscr{K}$. Let $p:[0,1] \rightarrow[0,1]$ be a map strictly controlled by $\mathscr{K}$. Suppose also that $(S, I, \pi)$ is a strip and $h_{0}$ is a homeomorphism of $[0,1]$ into the interior of $I$. Then there is an embedding $h$ of $[0,1]$ into the interior of $S$ so that $h(\mathscr{K})$ is $\pi$-exposed in $h([0,1])$ and $\pi(h(t))=h_{0}(p(t))$ for each $t \in[0,1]$.

Proof of Lemma 5.2. Without loss of generality we can assume that $S=[0,1] \times$ $[-2,2], I=\{0\} \times[-2,2], \pi$ is the projection of $S$ onto $I$, and $h_{0}(t)=(0, t)$ for each $t \in[0,1]$. We will show that there is a map $g:[0,1] \rightarrow[0,1)$ so that $h:[0,1] \rightarrow S$ defined by the formula $h(t)=(g(t), p(t))$ has the required properties.

Let $\mathscr{M}(\mathscr{K})$ be the collection of all minimal (under inclusion) elements of $\mathscr{K}$. We will define $g$ gradually on elements of $\mathscr{K}$ starting from $\mathscr{M}(\mathscr{K})$ and then extending it to other elements of $\mathscr{K}$. Let $g(t)=1 / 4+t / 4$ for each $t \in(\mathscr{M}(\mathscr{K}))^{*}$. To complete the proof of the lemma it is enough to extend $g$ to some $C \in \mathscr{K}$ assuming that it is already defined on all elements of $\mathscr{K}(C)$. 


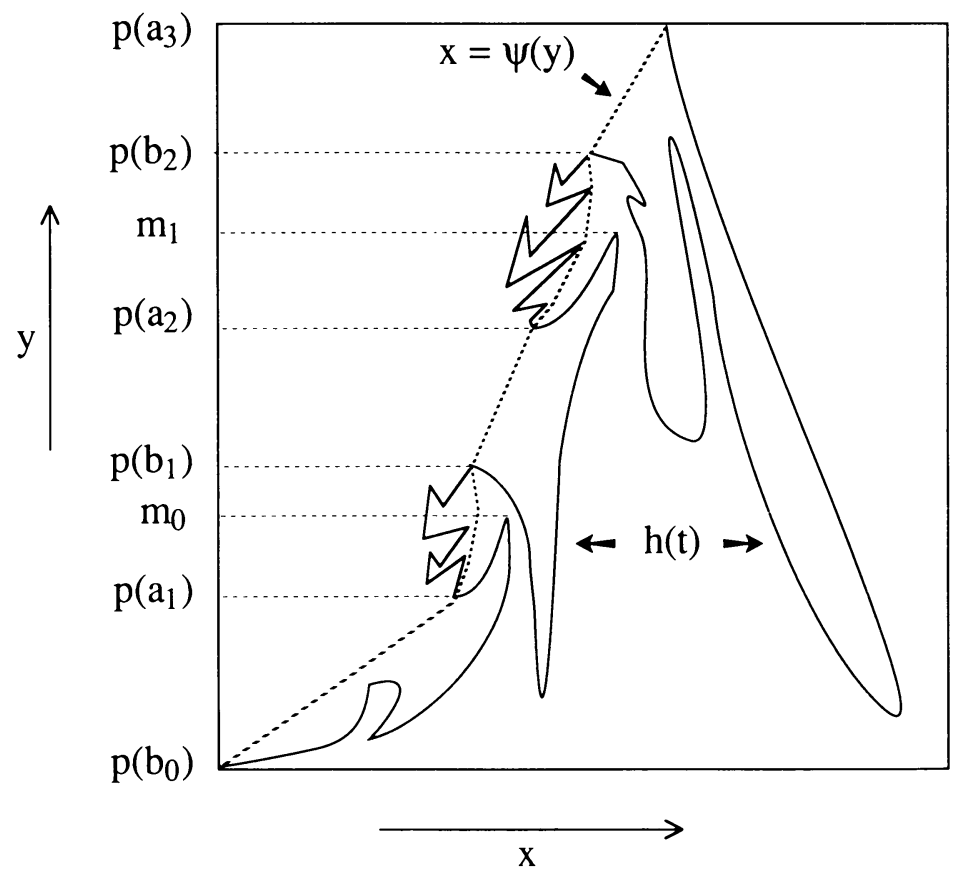

FIGURE 2

Let $C_{1}, C_{2}, \ldots, C_{n}$ be all the elements of $\mathscr{K}(C)$ ordered so that if $C_{i}=$ $\left[a_{i}, b_{i}\right]$ then $a_{1} \leq b_{1}<a_{2} \leq b_{2} \cdots<a_{n} \leq b_{n}$. We can assume that there is $c \in(0,1)$ so that $g\left(C_{i}\right) \subset[0, c]$ for each $i=1, \ldots, n$. Let $b_{0}$ denote the left end of $C$ and let $a_{n+1}$ denote the right end of $C$. Since $p$ is strictly controlled by $\mathscr{K}$ we have that either

(*) $p\left(\left[b_{0}, a_{i}\right]\right) \subset\left[p\left(b_{0}\right), p\left(b_{i}\right)\right)$ or

(**) $n=1$ and $p\left(\left[b_{1}, a_{2}\right]\right) \subset\left(p\left(a_{1}\right), p\left(a_{2}\right)\right]$.

The proof in case $(* *)$ is exactly the same as in case $(*)$ and $n=1$. So we can assume that $(*)$ holds.

Denote by $H_{i}$ the convex hull of $h\left(\left[a_{i}, b_{i}\right]\right)$. For each $t \in\left[p\left(a_{i}\right), p\left(b_{i}\right)\right]$ let $\psi_{i}(t)$ be the greatest real number so that $\left(\psi_{i}(t), t\right)$ is a point of $H_{i} \cap[0,1] \times$ $\{t\}$. Observe that $\psi_{i}$ is a continuous function of $\left[p\left(a_{i}\right), p\left(b_{i}\right)\right]$ and since $p\left(\left(a_{i}, b_{i}\right)\right) \subset\left(p\left(a_{i}\right), p\left(b_{i}\right)\right)$ we have that $\psi_{i}\left(p\left(a_{i}\right)\right)=g\left(a_{i}\right)$ and $\psi_{i}\left(p\left(b_{i}\right)\right)=$ $g\left(b_{i}\right)$. There is a map $\psi:\left[p\left(b_{0}\right), p\left(a_{n+1}\right)\right] \rightarrow[0, c]$ so that $\psi(t)=\psi_{i}(t)$ for each $t \in\left[p\left(a_{i}\right), p\left(b_{i}\right)\right]$ and $i=1, \ldots, n$.

Observe that since $p$ strictly respects the order of $\mathscr{K}, p\left(b_{0}\right)<p(t)<p\left(a_{n+1}\right)$ for each $t \in\left(b_{0}, a_{n+1}\right)$. If $b_{0}<a_{1}$, there is $a_{0}^{\prime} \in\left(b_{0}, a_{1}\right)$ so that $p(t)<p\left(a_{1}\right)$ for each $t \in\left[b_{0}, a_{0}^{\prime}\right]$. If $b_{n}<a_{n+1}$, there is $b_{n}^{\prime} \in\left(b_{n}, a_{n+1}\right)$ so that $p(t)>$ $p\left(a_{n}\right)$ for each $t \in\left[b_{n}^{\prime}, a_{n+1}\right]$. Define $b_{0}^{\prime}=b_{0}$ and $a_{n}^{\prime}=a_{n+1}$. If $n>1$ then by (iii) of the definition of $p$ being strictly controlled by $\mathscr{K}$ we have that for $i=1, \ldots, n-1$, there are $b_{i}^{\prime}$ and $a_{i}^{\prime}$ so that $b_{i}<b_{i}^{\prime}<a_{i}^{\prime}<a_{i+1}, p(t)<p\left(a_{i+1}\right)$ for each $t \in\left[b_{i}, a_{i}^{\prime}\right]$ and $p(t)>p\left(b_{i}\right)$ for $t \in\left[b_{i}^{\prime}, a_{i+1}\right]$. Applying Lemma 5.1 with $b=b_{i}, b^{\prime}=b_{i}^{\prime}, a^{\prime}=a_{i}^{\prime}$ and $a=a_{i+1}$ for each $i=0,1, \ldots, n$, we get a map $s_{i}:\left[b_{i}, a_{i+1}\right] \rightarrow[0,1]$ so that $s_{i}\left(b_{i}\right)=0, s_{i}\left(a_{i+1}\right)=0, s_{i}(t)>0$ for $b_{i}<t<a_{i+1}$, and the map $h_{i}:\left[b_{i}, a_{i+1}\right] \rightarrow S$ defined by the formula $h_{i}(t)=\left(s_{i}(t), p(t)\right)$ is an embedding of $\left[b_{i}, a_{i+1}\right]$ into $S$. 
Let $m_{i}$ be the maximum of $p$ on $\left[b_{i}, a_{i+1}\right]$. Note that $m_{i}<p\left(b_{i+1}\right)$, since $p$ satisfies $(*)$.

For each $i=0, \ldots, n-1$ there is a positive number $c_{i}<s_{i}(t)$ for each $t \in\left[b_{i+1}, a_{i+2}\right]$ with $p(t) \leq m_{i}$. Let $w_{n}$ be a positive constant less than $1-c$. For each $i=n-1, \ldots, 0$ let $w_{i}=w_{i+1} c_{i}$.

Extend $g$ to $\left[b_{0}, a_{n+1}\right]$ by setting $g(t)=\psi(p(t))+w_{i} s_{i}(t)$ for $t \in\left(b_{i}, a_{i+1}\right)$ and $i=0,1, \ldots, n$. Since $g(t)>\psi(p(t))$ for $t \in\left(b_{i}, a_{i+1}\right)$ and $i=$ $0,1, \ldots, n$, we have that $h\left(C_{i}\right)$ is $\pi$-exposed in $h(C)$. It may be verified that the construction of $g$ was so as to make $h$ an embedding.

Lemma 5.3. Suppose $\left(S_{0}, I_{0}, \pi_{0}\right),\left(S_{1}, I_{1}, \pi_{1}\right),\left(S_{2}, I_{2}, \pi_{2}\right), \ldots$ is a sequence of strips so that $\sum_{i=0}^{\infty} w\left(\pi_{i}\right)<\infty$ and $S_{i+1} \subset \operatorname{int}\left(S_{i}\right)$ for each positive integer $i$. Let $S=\bigcap_{i=0}^{\infty} S_{i}$. For each $i=0,1, \ldots$, let $D_{i} \subset I_{i}$ be an arc so that $D_{i+1}$ is $\pi_{i}$-exposed in $S_{i+1}$. Suppose the sequence $D_{0}, D_{1}, D_{2}, \ldots$ has a limit, $D$.

(i) If $\pi_{i}\left(D_{i+1}\right) \subset D_{i}$ for each $i=0,1, \ldots$ and $D$ is a one point set, then $D$ is accessible from the complement of $S$.

(ii) If $\pi_{i}\left(D_{i+1}\right)=D_{i}$ for each $i=0,1, \ldots$, then the set of points of $D$ which are accessible from the complement of $S$ is dense in $D$ and contains all points of local connectivity of $D$.

Proof of Lemma 5.3. Let $d$ be a point of $D$ and let $d_{i} \in D_{i}$ be so that $\lim _{i \rightarrow \infty} d_{i}=d$. Denote by $a_{i-1}$ the point $\pi_{i-1}\left(d_{i}\right)$. Let $A_{i}$ be the subarc of $F\left(\pi_{i-1}, d_{i}\right)$ containing $a_{i-1}$ so that the other endpoint $b_{i}$ of $A_{i}$ is the only point of the set $A_{i} \cap S_{i}$. Observe that $b_{i} \in D_{i}$, since $D_{i}$ is $\pi_{i-1}$-exposed in $S_{i}$. Since $\operatorname{diam}\left(F\left(\pi_{i-1}, d_{i}\right)\right) \leq w\left(\pi_{i-1}\right)$ and $\lim _{i \rightarrow \infty} w\left(\pi_{i}\right)=0$, we have that $\lim _{i \rightarrow \infty} A_{i}=\{d\}$ and in particular $\lim _{i \rightarrow \infty} a_{i}=\lim _{i \rightarrow \infty} b_{i}=d$. Let $B_{i}$ be the subarc of $I_{i}$ joining $a_{i}$ with $b_{i}$. Note that in both cases (i) and (ii) we have that $B_{i} \subset D_{i}, A_{i}$ and $B_{i}$ are both in the complement of $S, b_{i} \in A_{i} \cap B_{i}$ and $a_{i} \in B_{i} \cap A_{i+1}$. To show that $\{d\} \cup \bigcup_{i=1}^{\infty}\left(A_{i} \cup B_{i}\right)$ is an arc it is enough to show that $\lim _{i \rightarrow \infty} \operatorname{diam}\left(B_{i}\right)=0$. It is obvious in case (i). We prove it later in case (ii) if $D$ is locally connected at $d$.

Suppose $\pi_{i}\left(D_{i+1}\right)=D_{i}$ for each $i=0,1, \ldots$. For each point $c \in D_{i}$, let $\Phi(c)$ be the subarc of $F\left(\pi_{i}, c\right)$ containing $c$ so that the other endpoint $\varphi(c)$ of $\Phi(c)$ is the only point of the set $\Phi(c) \cap S_{i+1}$. Since $\pi_{i}\left(D_{i+1}\right)=D_{i}$ and $D_{i+1}$ is $\pi_{i}$-exposed in $S_{i+1}$, we have that $\varphi(c) \in D_{i+1}$ and the function $\varphi$ can be iterated. Since $\sum_{j=0}^{\infty} w\left(\pi_{j}\right)<\infty$, there is a point $\zeta(c) \in D$ so that the set $L(c)=\{\zeta(c)\} \cup \Phi(c) \cup \Phi(\varphi(c)) \cup \Phi\left(\varphi^{2}(c)\right) \cup \Phi\left(\varphi^{3}(c)\right) \cup \cdots$ is an arc. Note that $L(c) \cap S=\zeta(c)$ and $\operatorname{diam}(L(c)) \leq \sum_{j=1}^{\infty} w\left(\pi_{j}\right)$ if $c \in D_{i}$. It follows that the set $\bigcup_{i=0}^{\infty} \zeta\left(D_{i}\right)$, which consists of points accessible from the complement of $S$, is dense in $D$.

To complete the proof we have to show that $\lim _{i \rightarrow \infty} \operatorname{diam}\left(B_{i}\right)=0$. Let $\varepsilon$ be an arbitrary positive number. We will prove that $\operatorname{diam}\left(B_{i}\right)<\varepsilon$ for sufficiently large $i$. Since $D$ is locally connected at $d$, there is a continuum $V \subset D$ so that $d \in \operatorname{int}_{D}(V)$ and $\operatorname{diam}(V)<\varepsilon / 5$. Let $\delta>\varepsilon / 5$ be a positive number so that $\mathscr{B}(d, 2 \delta) \cap D \subset V$. Take $i$ so large that $a_{i}, b_{i} \in \mathscr{B}(d, \delta)$ and $\sum_{j=1}^{\infty} w\left(\pi_{j}\right)<$ $\delta$. It follows that $L\left(a_{i}\right) \cup L\left(b_{i}\right) \subset \mathscr{B}(d, 2 \delta)$ and consequently $\zeta\left(a_{i}\right)$ and $\zeta\left(b_{i}\right)$ belong to $V$. Observe that $C=L\left(a_{i}\right) \cup L\left(b_{i}\right) \cup V$ is a subcontinuum of $S_{i}$ so that $\operatorname{diam}(C)<2 \delta+\operatorname{diam}(V)<3 \varepsilon / 5$. Since $\left|\pi_{i}(s)-s\right| \leq w\left(\pi_{i}\right)<\delta$ for each $s \in S_{i}$, we have that $\operatorname{diam}\left(\pi_{i}(C)\right) \leq 2 \delta+\operatorname{diam}(C)<\varepsilon$. Since $a_{i}$ and $b_{i}$ 
are contained in $\pi_{i}(C)$ and $\pi_{i}(C)$ is connected, we have that $B_{i} \subset \pi_{i}(C)$ and consequently $\operatorname{diam}\left(B_{i}\right)<\varepsilon$. This completes the proof of the lemma.

\section{MAIN RESUltS}

Theorem 6.1. Suppose $X$ is an hereditarily decomposable chainable continuum and $\mathscr{Z}$ is a countable collection of generalized layers of $X$. Then there is an embedding $g$ of $X$ into the plane so that

(i) $\operatorname{Acc}(g(X)) \cap g(Z)$ is dense in $g(Z)$ for each $Z \in \mathscr{Z}$ and

(ii) if a point $z$ is either a layer of an element of $\mathscr{Z}$ or it is the intersection of some elements of $\mathscr{Z}$, then $g(z) \in \operatorname{Acc}(g(X))$.

Proof of Theorem 6.1. Let $\mathscr{Z}=\left\{Z_{0}, Z_{1}, Z_{2}, \ldots\right\}$. We will construct:

(1) a decreasing sequence of positive numbers $1>\mu_{0}>\mu_{1}>\mu_{2}>\cdots$,

(2) a sequence of finite collections $\mathscr{K}_{0} \subset \mathscr{K}_{1} \subset \mathscr{K}_{2} \subset \cdots$ of generalized layers of $X$,

(3) a sequence of strips $\left(S_{0}, I_{0}, \pi_{0}\right),\left(S_{1}, I_{1}, \pi_{1}\right),\left(S_{2}, I_{2}, \pi_{2}\right), \ldots$, and

(4) a sequence of maps $g_{0}, g_{1}, g_{2}, \ldots$ so that

(i) $X, Z_{i} \in \mathscr{K}_{i}$ for each $i=0,1, \ldots$,

(ii) $g_{i}$ is a map from $X$ into the interior of $I_{i}$ so that it is controlled by $\mathscr{K}_{i}$ for each $i=0,1, \ldots$,

(iii) if $|x-y|>2^{-i}$ then $\left|g_{i}(x)-g_{i}(y)\right|>\mu_{i}$ for each $x, y \in X$ and each $i=0,1, \ldots$,

(iv) $w\left(\pi_{i}\right)<\mu_{i} 2^{-i-3}$ for each $i=0,1, \ldots$,

(v) $S_{i} \subset \operatorname{int}\left(S_{i-1}\right)$ for each $i=1,2, \ldots$,

(vi) $\pi_{i-1}\left(g_{i}(C)\right)=g_{i-1}(C)$ for each $C \in \mathscr{K}_{i-1}$ and $i=1,2, \ldots$,

(vii) $\pi_{i-1}$ exposes $g_{i}\left(\mathscr{K}_{i-1}\right)$ in $S_{i}$ for each $i=1,2, \ldots$, and

(viii) $\left|g_{i}(x)-g_{i-1}(x)\right|>\mu_{i-1} 2^{-i-1}$ for each $x \in X$ and $i=1,2, \ldots$.

Applying Lemma 4.4 with $\varepsilon=1$ and $\mathscr{Z}=\left\{X, Z_{0}\right\}$ we get a finite collection $\mathscr{K}_{0}$ and a 1 -map $f_{0}: X \rightarrow(0,1)$ so that $X, Z_{0} \in \mathscr{K}_{0}$ and $f_{0}$ is controlled by $\mathscr{K}_{0}$. Let $I_{0}$ be an arc lying in the plane and let $h_{0}$ be a homeomorphism of $[0,1]$ into $I_{0}$. Let $g_{0}=h_{0} \circ f_{0}$. Observe that $g_{0}: X \rightarrow I_{0}$ is a 1 -map which controlled by $\mathscr{K}_{0}$. There is a positive number $\mu_{0}<1$ so that if $|x-y| \geq 1$ then $\left|g_{0}(x)-g_{0}(y)\right|>\mu_{0}$ for each $x, y \in X$. Let $S_{0}$ be a disk containing $I_{0}$ in the boundary and let $\pi_{0}$ be a retraction of $S_{0}$ to $I_{0}$ so that $\left(S_{0}, I_{0}, \pi_{0}\right)$ is a strip and $w\left(\pi_{0}\right)<\mu_{0} 2^{-3}$.

Suppose $\mu_{i-1}, \mathscr{K}_{i-1},\left(S_{i-1}, I_{i-1}, \pi_{i-1}\right)$ and $g_{i-1}$ have been constructed. We will construct $\mu_{i}, \mathscr{K}_{i},\left(S_{i}, I_{i}, \pi_{i}\right)$ and $g_{i}$.

Let $h_{i-1}$ be a homeomorphism of $[0,1]$ onto $g_{i-1}(X)$ and let $f_{i-1}=h_{i-1}^{-1}$ 。 $g_{i-1}$. There is a positive number $\varepsilon$ so that if $|t-z|<\varepsilon$ then $\mid h_{i-1}(t)-$ $h_{i-1}(z) \mid<\mu_{i-1} 2^{-i-2}$ for each $t, z \in[0,1]$. Let $\delta$ be a number satisfying the conclusion of Lemma 4.5 applied with this $\varepsilon, f=f_{i-1}$ and $\mathscr{K}=\mathscr{K}_{i-1}$. We can assume that $\delta<2^{-i}$. Applying Lemma 4.4 we get a finite collection $\mathscr{K}_{i}$ and a $\delta$-map $f_{i}$ of $X$ onto $[0,1]$ so that $\mathscr{K}_{i-1} \cup\left\{Z_{i}\right\} \subset \mathscr{K}_{i}$ and $f_{i}$ is controlled by $\mathscr{K}_{i}$. Observe that $f_{i}$ respects the order of $\mathscr{K}_{i-1}$, since it respects the order of $\mathscr{K}_{i}$ and $\mathscr{K}_{i-1} \subset \mathscr{K}_{i}$. By the choice of $\delta$, there is a map $p:[0,1] \rightarrow[0,1]$ so that $p$ is strictly controlled by $f_{i}\left(\mathscr{K}_{i-1}\right), p\left(f_{i}(e)\right)=f_{i-1}(e)$ for each $e \in E\left(\mathscr{K}_{i-1}\right)$ and $\left|p\left(f_{i}(x)\right)-f_{i-1}(x)\right|<\varepsilon$ for each $x \in X$. Applying Lemma 5.2 we get an embedding $h_{i}$ of $[0,1]$ into the interior of $S_{i-1}$ so that $h_{i}\left(f_{i}\left(\mathscr{K}_{i-1}\right)\right)$ is 
$\pi_{i-1}$-exposed in $h_{i}([0,1])$ and $\pi_{i-1}\left(h_{i}(t)\right)=h_{i-1}(p(t))$ for each $t \in[0,1]$. Observe that $\pi_{i-1}\left(h_{i}(0)\right)$ and $\pi_{i-1}\left(h_{i}(1)\right)$ are the ends of $\pi_{i-1}\left(h_{i}([0,1])\right)$. The arc $h_{i}([0,1])$ can be extended to an arc $I_{i}$ containing $h_{i}([0,1])$ in its interior so that $I_{i} \subset \operatorname{int}\left(S_{i}\right)$, the ends of $I_{i}$ are mapped by $\pi_{i-1}$ onto the ends of $\pi_{i-1}\left(I_{i}\right)$ and $h_{i}\left(f_{i}\left(\mathscr{K}_{i-1}\right)\right)$ is $\pi_{i-1}$-exposed in $I_{i}$. Let $g_{i}=h_{i} f_{i}$. Observe that $g_{i}$ is a $\delta$-map controlled by $\mathscr{K}_{i}$ and that $\pi_{i-1}\left(g_{i}(C)\right)=g_{i-1}(C)$ for each $C \in \mathscr{K}_{i-1}$. Since $\delta<2^{-i}$, there is a positive number $\mu_{i}<\mu_{i-1}$ so that if $|x-y| \geq 2^{-i}$ then $\left|g_{i}(x)-g_{i}(y)\right|>\mu_{i}$ for each $x, y \in X$. Since the ends of $I_{i}$ are mapped by $\pi_{i-1}$ onto the ends of $\pi_{i-1}\left(I_{i}\right)$, there is a disk $S \subset \operatorname{int}\left(S_{i-1}\right)$ containing $I_{i}$ in its boundary so that $\left\{I_{i}\right\}$ is $\pi_{i-1}$-exposed in $S$. There is a projection $\pi$ of $S$ to $I_{i}$ so that $\left(S, I_{i}, \pi\right)$ is a strip. By taking a disk $S_{i}$ close to $I_{i}$ and defining $\pi_{i}$ to be $\pi$ restricted to $S_{i}$ we can get a strip $\left(S_{i}, I_{i}, \pi_{i}\right)$ with $w\left(\pi_{i}\right)<\mu_{i} 2^{-i-3}$. It is easy to verify conditions (i) through (vii) of the construction. We will prove here condition (viii).

Let $x$ be an arbitrary point of $X$. Since $\left|p\left(f_{i}(x)\right)-f_{i-1}(x)\right|<\varepsilon$, by the choice of $\varepsilon$ we have that

$$
\left|h_{i-1}\left(p\left(f_{i}(x)\right)\right)-h_{i-1}\left(f_{i-1}(x)\right)\right|<\mu_{i-1} 2^{-i-2} .
$$

Since $\pi_{i-1}\left(h_{i}\left(f_{i}(x)\right)\right)=h_{i-1}\left(p\left(f_{i}(x)\right)\right)$ and $w\left(\pi_{i-1}\right)<\mu_{i-1} 2^{-i-2}$, we have that

$$
\left|h_{i}\left(f_{i}(x)\right)-h_{i-1}\left(p\left(f_{i}(x)\right)\right)\right|<\mu_{i-1} 2^{-i-2},
$$

and consequently

$$
\left|g_{i}(x)-g_{i-1}(x)\right|=\left|h_{i}\left(f_{i}(x)\right)-h_{i-1}\left(f_{i-1}(x)\right)\right|<\mu_{i-1} 2^{-i-1} .
$$

So the construction is complete.

It follows from (viii) that $\left|g_{k}(x)-g_{i}(x)\right|<\mu_{i} 2^{-i+1}$ if $0 \leq i<k$ and $x \in X$. So the sequence $\left\{g_{i}\right\}$ converges uniformly to a continuous map $g: X \rightarrow$ $\bigcap_{i=0}^{\infty} S_{i}$. Note that $\left|g(x)-g_{i}(x)\right| \leq \mu_{i} 2^{-i+1}$ for each $i=0,1, \ldots$ and $x \in X$. For each two different points $x$ and $y$ from $X$ there is a positive integer $i$ so that $|x-y|>2^{-i}$. Then $\left|g_{i}(x)-g_{i}(y)\right|>\mu_{i}$, by (iii). It follows that $|g(x)-g(y)|>\mu_{i}-2 \mu_{i} 2^{-i+1}>0$ for $i>2$, so $g(x) \neq g(y)$ if $x \neq y$. This proves that $g$ is an embedding.

Observe that if $C$ is the intersection of a decreasing sequence $C_{0}, C_{1}, C_{2}$, .. of subcontinua of $X$, then $\lim _{i \rightarrow \infty} g_{i}\left(C_{i}\right)=g(C)$.

Let a point $z$ be the intersection of some elements of $\mathscr{Z}$. Let $C_{i}$ be the minimal element of $\mathscr{K}_{i}$ containing $z$. Then $\{z\}=\bigcap_{i=0}^{\infty} C_{i}$. Applying Lemma 5.3 with $D_{i}=g\left(C_{i}\right)$ we get that $g(z)$ is accessible from the complement of $g(X)$.

Let $Z$ be an element of $\mathscr{Z}$. There is a positive integer $j$ so that $Z \in \mathscr{K}_{i}$ for each $i \geq j$. Applying Lemma 5.3 with $D_{i}=g(Z)$ we get that $\operatorname{Acc}(g(X)) \cap g(Z)$ is dense in $g(Z)$ and contains all points of local connectivity of $g(Z)$. Since an hereditarily decomposable chainable continuum $Z$ is locally connected at its point $z$ if and only if $z$ is a degenerate layer of $Z$, the proof of the theorem is complete.

The proofs of the Lemmas 6.2 and 6.4 use a technique similar to that in [5, 9 and 12].

Lemma 6.2. Suppose $X$ is a plane compactum and $\mathscr{K}$ is a collection of mutually exclusive nondegenerate continua contained in $X$. If each point of $\mathscr{K}^{*}$ is accessible from the complement of $X$, then $\mathscr{K}$ is countable. 
Proof of Lemma 6.2. Suppose that $\mathscr{K}$ is uncountable. For each $C \in \mathscr{K}$ let $a_{C}$ and $b_{C}$ be two points accessible from the complement of $X$. Without loss of generality we may assume that $C$ is irreducible between $a_{C}$ and $b_{C}$. There exist disjoint disks $D_{a}$ and $D_{b}$ so that $a_{C}$ and $b_{C}$ are contained in $D_{a}$ and $D_{b}$, respectively for each $C$ belonging to an uncountable subcollection of $\mathscr{K}$, which we can assume is $\mathscr{K}$ itself.

Let $F_{C} \subset C$ be a continuum irreducibly intersecting both $D_{a}$ and $D_{b}$. A point of $F_{C} \backslash\left(D_{a} \cup D_{b}\right)$ is accessible from some component $G_{C}$ of the complement of $X \cup D_{a} \cup D_{b}$. So there is a component $G_{C}$ of the complement of $X \cup D_{a} \cup D_{b}$ so that $\operatorname{cl}\left(G_{C}\right)$ intersects $F_{C}$. Since no component of the complement of $X \cup D_{a} \cup D_{b}$ can intersect more than two sets $F_{C}$ and there are only countably many components of the complement of $X \cup D_{a} \cup D_{b}, \mathscr{K}$ could not have been uncountable.

The following corollary answers Problem 6 from [11].

Theorem 6.3. A chainable continuum $X$ can be embedded in the plane in such a way that every point is accessible from its complement if and only if it is Suslinean.

Proof of Theorem 6.3. It was observed by A. Lelek (see Comment to Problem 6 in [11]) that if $X$ is not Suslinean then, regardless of its embedding in the plane, it has inaccessible points (or this may be deduced from Lemma 6.2). If $X$ is Suslinean then $X$ is hereditarily decomposable. Let $\mathscr{Z}$ be the collection of all nondegenerate generalized layers of $X$. Since $X$ is Suslinean this collection is countable. Since each point of $X$ is a generalized layer of $X$, each point of $X$ is either a layer of an element of $\mathscr{Z}$ or the intersection of such. Now we can apply Theorem 6.1 , thereby obtaining an embedding with all points accessible.

Lemma 6.4. Suppose $X$ is a chainable hereditarily decomposable continuum contained in the plane. Let $S$ be a connected dense subset of $X$ and let $\mathscr{K}$ be a collection of mutually exclusive nondegenerate continua contained in $X \backslash S$. If each element of $\mathscr{K}$ contains at least two points accessible from the complement of $X$, then $\mathscr{K}$ is countable.

Proof of Lemma 6.4. Suppose that $\mathscr{K}$ is uncountable. For each $C \in \mathscr{K}$ let $a_{C}$ and $b_{C}$ be two points accessible from the complement of $X$. Without loss of generality we may assume that $C$ is irreducible between $a_{C}$ and $b_{C}$. Since $X$ does not separate the plane, there is an arc $L_{C}$ contained in the plane meeting $X$ only at the points $a_{C}$ and $b_{C}$ which are its ends. Observe that $C \cup L_{C}$ separates the plane into two components. Since $S$ is dense in $X$ and $S \cap\left(C \cup L_{C}\right)=\varnothing$, one of the components of the complement of $C \cup L_{C}$ does not intersect $X$. We will denote this component by $A_{C}$. In each $C$ choose a point $z_{C}$ different from both $a_{C}$ and $b_{C}$ and let $\delta_{C}$ be the distance of $z_{C}$ to $L_{C}$. Since $\delta_{C}>0$ for each $C \in \mathscr{K}$, there is a positive number $\varepsilon$ and an uncountable collection $\mathscr{K}_{0} \subset \mathscr{K}$ so that $\delta_{C}>\varepsilon$ for each $C \in \mathscr{K}_{0}$. There is an uncountable collection $\mathscr{K}_{1} \subset \mathscr{K}_{0}$ and an open disk $D$ so that $\operatorname{diam}(D)<\varepsilon$ and $z_{C} \in D$ for each $C \in \mathscr{K}_{1}$. Observe that since $C$ is irreducible, $D \cap A_{C} \neq \varnothing$. For each $C \in \mathscr{K}_{1}$ choose a component $E_{C}$ of $D \cap A_{C}$.

Suppose $E_{C} \cap E_{C^{\prime}} \neq \varnothing$ for any two different $C, C^{\prime} \in \mathscr{K}_{1}$. Then $\operatorname{cl}\left(E_{C^{\prime}}\right) \subset$ $A_{C}$, but since $\operatorname{cl}\left(E_{C^{\prime}}\right) \cap C^{\prime} \neq \varnothing, C^{\prime}$ would intersect $A_{C}$ which is a contradic- 
tion. So $\left\{E_{C} \mid C \in \mathscr{K}_{1}\right\}$ is an uncountable collection of mutually exclusive open sets in the plane. This contradiction completes the proof of the lemma.

Theorem 6.5. An hereditarily decomposable chainable continuum $X$ can be embedded in the plane in such a way that each endpoint is accessible from the complement if and only if there are only countably many nondegenerate generalized end layers.

Proof of Theorem 6.5. Let $\mathscr{Z}$ be the collection of nondegenerate generalized end layers.

If $\mathscr{Z}$ is uncountable then it contains an uncountable subcollection $\mathscr{K}$ of mutually disjoint nondegenerate generalized end layers. Since each element of $\mathscr{K}$ contains at least two endpoints it follows from Lemma 6.4 that $X$ cannot be embedded in such a way that each endpoint is accessible from the complement of $X$.

If $\mathscr{Z}$ is countable then each endpoint is either a layer of an element of $\mathscr{Z}$ or it is the intersection of some elements of $\mathscr{Z}$, therefore it follows from Theorem 6.1 that $X$ can be embedded in such a way that each endpoint is accessible from the complement of $X$.

In view of Example 3.1 Theorem 6.5 answers Problem 5 from [11].

Corollary 6.6. The hereditarily decomposable chainable continuum in Example 3.1 cannot be embedded in such a way that each endpoint is accessible from the complement.

\section{REFERENCES}

1. R. H. Bing, Snake-line continua, Duke Math. J. 18 (1951), 653-663.

2. J. B. Fugate, Decomposable chainable continua, Trans. Amer. Math. Soc. 123 (1966), 460468.

3. __ A characterization of chainable continua, Canad. J. Math. 21 (1969), 383-393.

4. K. Kuratowski, Topology, Vol. II, Academic Press, New York and Polish Scientific Publishers, Warsaw, 1968.

5. A. Lelek, On the Moore triodic theorem, Bull. Acad. Polon. Sci. 7 (1960), 271-276.

6. S. Mazurkiewicz, Sur les points accessibles des continus indécomposables, Fund. Math. 14 (1929), 107-115.

7. P. Minc and W. R. R. Transue, Sarkovskii's theorem for hereditarily decomposable chainable continua, Trans. Amer. Math. Soc. 315 (1989), 173-188.

8. L. Mohler, The depth of tranches in ג-dendroids, Proc. Amer. Math. Soc. 96 (1986), 715720.

9. R. L. Moore, Concerning triodic continua in the plane, Fund. Math. 13 (1929), 261-263.

10. E. S. Thomas, Jr., Monotone decompositions of irreducible continua, Rozprawy Mat. 50 (1960).

11. University of Houston Problem Book, (mimeographed notes).

12. G. S. Young, Jr., A generalization of Moore's theorem on simple triods, Bull. Amer. Math. Soc. 50 (1944), 714.

fat Mathematics, Auburn University, Auburn, Alabama 36849

E-mail address: pminc@ducvax.auburn.edu 